\title{
Conduction and electric field effect in ultra-thin tungsten films
}

\author{
Kees van der Zouw, Antonius A.I. Aarnink, Jurriaan Schmitz, Alexey Y. Kovalgin \\ MESA+ Institute for Nanotechnology, University of Twente, P.O. Box 217, 7500 AE, Enschede, The Netherlands \\ Email: k.vanderzouw@utwente.nl
}

\begin{abstract}
Ultra-thin tungsten films were prepared using hotwire assisted atomic layer deposition. The film thickness ranged from 2.5 to $10 \mathrm{~nm}$, as determined by spectroscopic ellipsometry and verified by scanning electron microscopy. The films were implemented in conventional Van der Pauw and circular transmission line method (CTLM) test structures, to explore the effect of film thickness on the sheet and contact resistance, temperature coefficient of resistance (TCR), and external electric field applied. All films exhibited linear current-voltage characteristics. The sheet resistance was shown to considerably vary across the wafer, due to the film thickness non-uniformity. The TCR values changed from positive to negative with decreasing the film thickness. A field-induced modulation of the sheet resistance up to $\sim 4.6 \cdot 10^{-4} \mathrm{~V}^{-1}$ was obtained for a $2.5 \mathrm{~nm}$ thick film, larger than that generally observed for metals.

Index Terms-Thin films, tungsten, hot-wire, atomic layer deposition, spectroscopic ellipsometry, scanning electron microscopy, X-ray diffraction, sheet resistance, contact resistance, transfer length, temperature coefficient of resistance, field effect
\end{abstract}

\section{INTRODUCTION}

The continuous downscaling of electronic devices poses an increasing demand for the use of ultra-thin films in a variety of applications such as microprocessors, image sensors, memories, and physical unclonable-function devices $[1,2]$. Both isolating and metallic layers are widely requested. Among a variety of characteristics, film conformality, uniformity, thickness, step coverage and resistivity may be of crucial importance. Tungsten (W) is one of the metals commonly adopted by industry in integrated circuits to realize electrodes and interconnects [3]. With decreasing the film thickness, the main challenge is to keep the sheet and contact resistances low enough, in line with the application demands [4]. Further, the ultra-thin metal films may be expected to exhibit a field effect, which is not observable in the thick layers due to the high electron density and the related screening effect [5-10]. All this makes determining the electrical behavior of ultra-thin $\mathrm{W}$ films relevant and important.

Atomic Layer Deposition (ALD) is a deposition technique that perfectly fits with the need for miniaturization. ALD is known to provide high layer uniformity and conformality, together with excellent step-coverage and precise layerthickness control, due to its attribute of sequential, selflimiting surface reactions [11]. This makes ALD the method of choice for many applications. ALD can be categorized in two main classes: thermal ALD and radical enhanced ALD (REALD). Many single element deposition processes can not be executed in pure thermal mode $[11,12]$. Radicals enable reactions which would otherwise not occur. A plasma is often used as a source of radicals. However, there is a number of drawbacks while using a plasma. First, a plasma can damage the wafer. Secondly, multiple reactions take place in plasma: the wafer may be exposed to unwanted radicals, atoms, ions, or UV photons [13]. Recently, a novel approach to ALD was developed, the so-called hot-wire assisted ALD (HWALD) [14]. A hot-wire takes the role of the plasma as the radical source. HWALD has been successfully applied to deposit either $\alpha$-or $\beta$-phase crystalline $\mathrm{W}$, depending on the conditions [15-19].

In this work, we applied conventional Van der Pauw and circular transmission line method (CTLM) test structures, to explore the electrical properties of HWALD W films grown in the thickness range of $2.5-10 \mathrm{~nm}$. The influence of film thickness on the sheet and contact resistance, temperature coefficient of resistance (TCR) and external electric field effect (FE) were studied. This work extends our previous ICMTS 2019 publication [20] by adding a detailed analysis of the W-film thickness with spectroscopic ellipsometry (SE), further verified by high resolution scanning electron microscopy (SEM). X-ray diffraction (XRD) analysis of the crystal structures of HWALD W films is additionally provided.

\section{Test StRUCTURE FABRICATION}

Highly doped p-type 4-inch (100) Si wafers were used as substrates (see Fig. 1 for the process flow). Prior to thermal oxidation, the wafers were ozone-steam cleaned, followed by an $1 \% \mathrm{HF}$ deep for $1 \mathrm{~min}$. Oxidation was carried out at $1100{ }^{\circ} \mathrm{C}$ for 45 minutes in dry oxygen, to obtain approximately $100 \mathrm{~nm}$ of $\mathrm{SiO}_{2}$. Photoresist was applied and patterned according to the desired electrode shapes. Sputtering of a 10-nm-thick titanium adhesion layer and $40 \mathrm{~nm}$ thick platinum (Pt) layer was followed by a lift-off step to pattern the Pt electrodes. In our fabrication process, the electrodes are deposited before the to-be-analyzed $\mathrm{W}$ film, mainly to minimize processing on top of fragile ultra-thin layers. In electrical measurements, probes can easily pierce through the top layers to reach the electrodes. Next, a tungsten film and an a-Si capping layer (to prevent oxidation of the $\mathrm{W}$ in air) were deposited by HWALD and chemical vapor deposition 


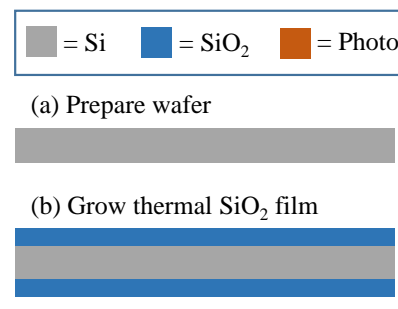

(c) Photolithography

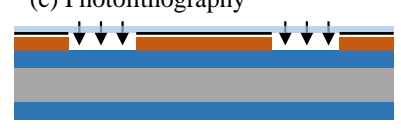

(d) Ti/Pt electrode sputtering

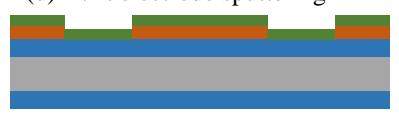

(e) Lift-off

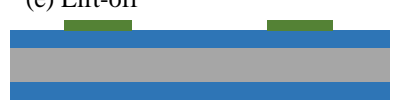

(f) Grow W film + a-Si capping

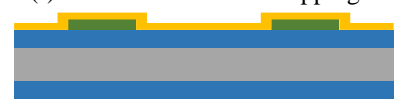

(g) Photolithography

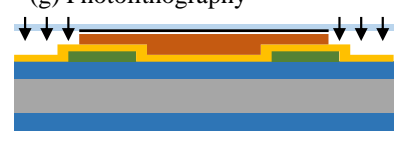

$=\mathrm{Ti} / \mathrm{Pt} \quad \mathrm{W}=\mathrm{W} / \mathrm{a}-\mathrm{Si} \quad \mathrm{Q}=\mathrm{Al}$

(h) W + a-Si wet etch

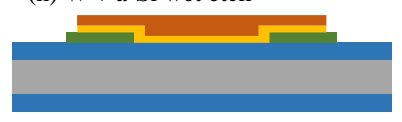

(i) Lift-off

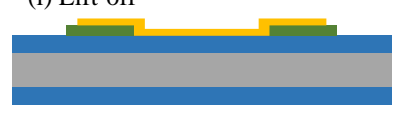

(j) Spin-coating of photoresist (to protect front-surface)

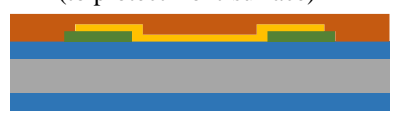

(k) Back-surface $\mathrm{SiO}_{2}$ wet etch

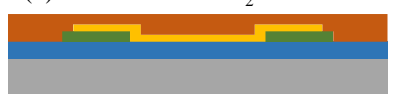

(1) Back-side Al electrode sputtering

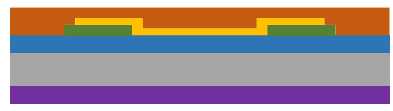

(m) Lift-off

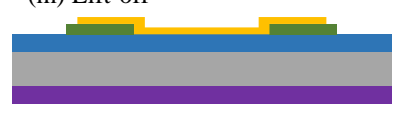

Fig. 1. Schematic of the process flow to fabricate the test structures. The process steps are treated in more detail in section II.

(CVD), respectively. Further patterning was performed by a second lithography step, subsequent wet-etching of the $\mathrm{W}$ and a-Si layers in a solution containing $0.67 \%$ of $\mathrm{HF}$ and $50 \%$ of $\mathrm{HNO}_{3}$, and by stripping the photoresist in fuming $\mathrm{HNO}_{3}$. A new layer of photoresist was applied to protect the front-surface during the back-side $\mathrm{SiO}_{2}$ removal in buffered HF. Sputtering of a 400-nm-thick aluminum film as the back-side (gate) electrode finalized the structures.

The HWALD deposition of $\mathrm{W}$ was performed from tungsten-hexafluoride $\left(\mathrm{WF}_{6}\right)$ and hydrogen $\left(\mathrm{H}_{2}\right)$ precursors at a substrate temperature of $275^{\circ} \mathrm{C}$. The hot-wire dissociates the $\mathrm{H}_{2}$ into atomic hydrogen (at-H) radicals. A hot-wall reactor was used to grow low-resistivity $\alpha$-phase $\mathrm{W}$ films [18]. No growth of $\mathrm{W}$ was observed on $\mathrm{SiO}_{2}$ directly [19]. To initiate the HWALD growth of $\mathrm{W}$, a CVD a-Si seed layer is grown on top of the $\mathrm{SiO}_{2}$ by dissociation of trisilane $\left(\mathrm{Si}_{3} \mathrm{H}_{8}\right)$, and subsequently converted into a $\mathrm{W}$ seed layer by introducing $\mathrm{WF}_{6}$ into the reactor.

\section{Test STRUCTURE DESIGN}

Each wafer is subdivided into process evaluation modules (PEMs). The PEM layout is shown in Fig. 2a. Each PEM consists of several Van der Pauw, CTLM, Greek-cross

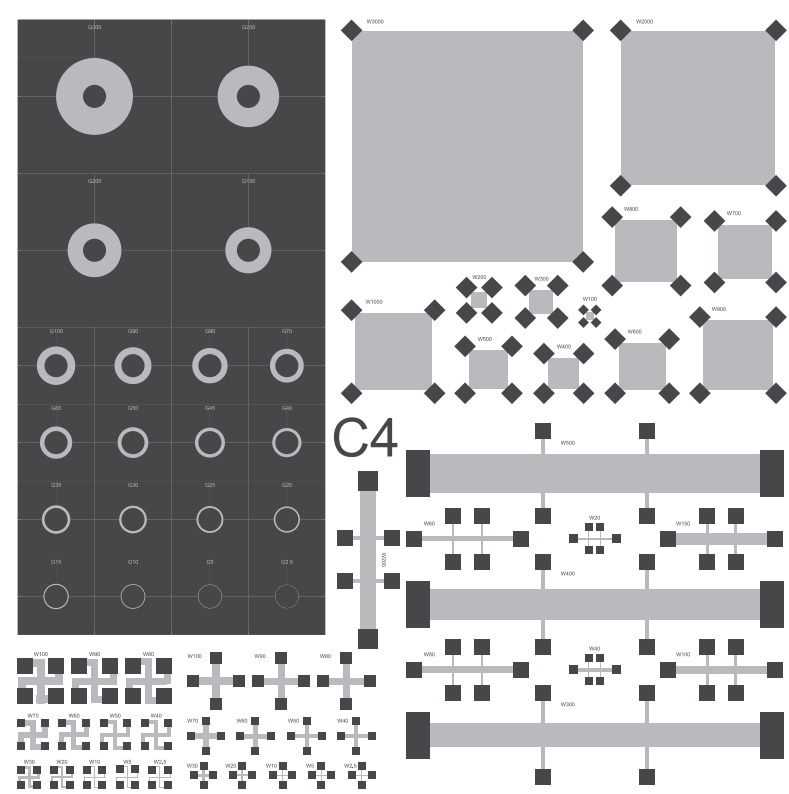

(a)

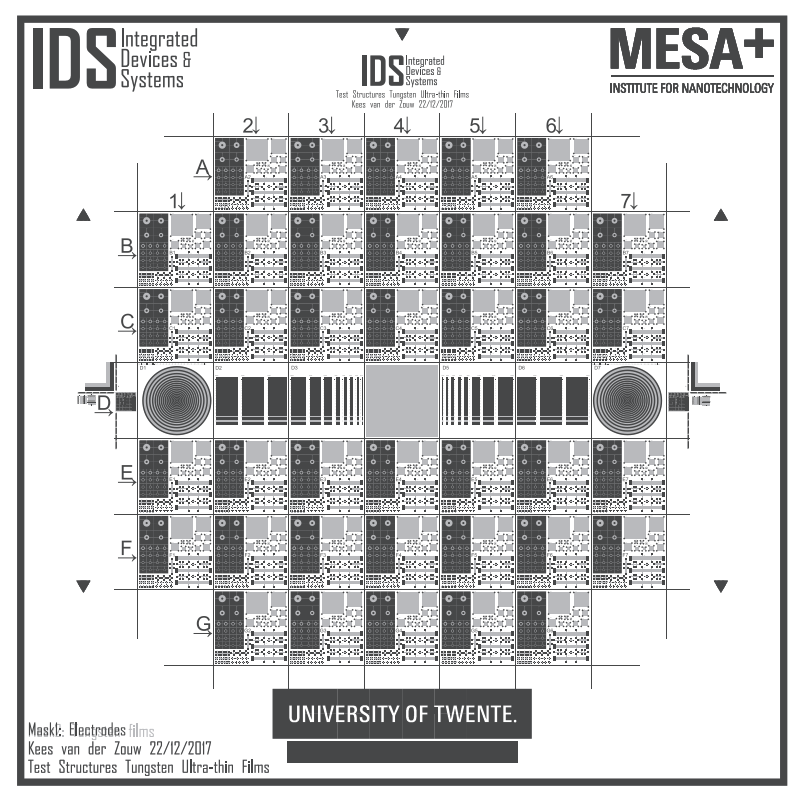

(b)

Fig. 2. (a) The process evaluation module with different test structures. (b) The wafer layout including all the modules.

and Hall structures of various dimensions. As shown in Fig. $2 b$, the PEMs were evenly distributed across the wafer surface.

For each type of structure, several issues were considered in the design process. Only the considerations for the Van der Pauw and CTLM structures will be discussed in detail, since all results presented in this work involve measurements on these structures. The Van der Pauw equation for the sheet resistance holds for the ideal case of infinitesimally small contacts at the circumference of the sample [21,22]. In practice, this condition is hardly met and a correction 

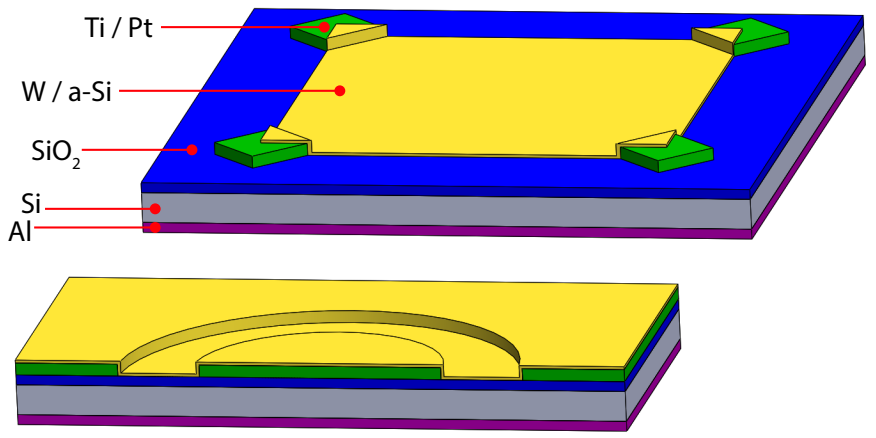

Fig. 3. Schematic representation of the typical test structures used in this work. (Top) The Van der Pauw structure. (Bottom) the CTLM structure. The dimensions are not to scale.

factor should be included. The design of the Van der Pauw structures used in this work (see top of Fig. 3) is based on the symmetrical octagon structure that was analytically treated by [23], it also has electrodes located at the corners of the sheet. The ratio between the contact length and the total length of the structures at the circumference of the structure is kept around 0.1 for each structure, which means that the Van der Pauw equation should hold with high accuracy [23]. Small deviations of the electrode placement can be expected due to the alignment inaccuracy during fabrication. This was kept within $1 \mu \mathrm{m}$. Initial dual configuration measurements on the wafer with the $10-\mathrm{nm} \mathrm{W}$ film showed that the asymmetry in the measured sheet resistance $\left(R_{\mathrm{sh}}\right)$ was below $1 \%$ and therewith considered negligible. Small adaptations to the design can be applied to decrease sensitivity to alignment accuracy.

The CTLM structures, as designed for this work, have a circular inner electrode, separated from the outer electrode by a gap $W_{\mathrm{g}}$ (variable), as shown at the bottom of Fig. 3. By choosing a radius for the inner electrode $\left(R_{\mathrm{el}}\right)$ much larger than $W_{\mathrm{g}}$ and four times the transfer length $\left(L_{\mathrm{T}}\right)$, the values for $R_{\mathrm{sh}}, L_{\mathrm{T}}$ and the contact resistance $\left(R_{\mathrm{C}}\right)$ can be extracted from the corresponding resistance versus $W_{\mathrm{g}}$ plot $[24,25]$. It is assumed that the electrode resistance is negligible and the W sheet resistance on top of the electrodes and within the gap is identical [26,27]. The main difficulty in the design process of the CTLM structures is related to finding the $L_{\mathrm{T}}$. $L_{\mathrm{T}}$ is the length over which the electrical current is transferred between the electrodes and the $\mathrm{W}$ sheet. It can be extracted experimentally, however, an educated guess of its magnitude has to be made beforehand to meet the $R_{\mathrm{el}} \gg 4 L_{\mathrm{T}}$ condition. In this work, the $R_{\mathrm{el}}$ was chosen at $150 \mu \mathrm{m}$.

\section{OPTICAL MEASUREMENT RESULTS}

The thickness of the HWALD W was monitored in-situ by spectroscopic ellipsometry (SE). SE is generally used to determine the layer thickness $(t)$ and optical constants $\left(\epsilon_{1}, \epsilon_{2}\right)$. SE is a non-destructive optical characterization technique in which the change in polarization of a light beam upon reflection at the sample is monitored. The change in polarization is measured through the experimentally measured psi $(\Psi)$ and delta $(\Delta)$ parameters $[28,29]$. Especially $\Delta$ is a sensitive measure of the thickness of ultra-thin films. For the majority of samples, no simple relation exists between the measured $(\Psi, \Delta)$ and desired $\left(\epsilon_{1}, \epsilon_{2}, t\right)$ quantities. It is therefore necessary to propose a model that adequately describes $\epsilon_{1}, \epsilon_{2}$ and $t$ of all the layers and compare the model against the measurement results. The accuracy of the model is determined by the mean square error (MSE), which mathematically quantifies the difference between the experimental and model-generated data. In this work, the in-situ SE monitoring was performed by a Woollam M2000 spectroscopic ellipsometer operating in the wavelength range between 254 and $1688 \mathrm{~nm}$, in combination with COMPLETEEASE modeling software.

In this work, an optical model is proposed comprising of a silicon substrate, a thermally grown $\mathrm{SiO}_{2}$ layer and the HWALD W layer. The optical properties of Si [30] and $\mathrm{SiO}_{2}$ [31] are taken from the software database. In earlier research, the dielectric function of tungsten was determined for several wavelength ranges [32,33]. It was shown that the dielectric function can be parameterized by applying the Drude-Lorentz model. A Drude term describes the intraband absorption, whereas Lorentz oscillators describe the interband absorption by electrons [34,35]. Importantly, the optical properties of ultra-thin tungsten are unknown and expected to be significantly different from those of the bulk material [36-39]. Consequently, the optical properties have to be obtained through SE data fitting. The latter should be enabled by a scientifically correct model, in order to reliably extract a variety of physical properties.

For ultra-thin films, the refractive index and the thickness are likely correlated to each other in the model [28]. In other words, actual change of the physical thickness can be interpreted as a change of the refractive index; this significantly lowers the confidence on the calculated thickness values. For a multi-layer structure, fixing in the model both thickness and optical function values for the layers which are known, can significantly reduce the probability of such correlation. The $\mathrm{SiO}_{2}$ layer thickness was measured ex-situ before deposition. Together with the known optical functions, all $\mathrm{SiO}_{2}$ properties were further fixed in the optical model. The HWALD W thickness evolution was in-situ monitored, followed by ex-situ data processing for refining the extracted characteristics. Briefly, the layer of interest was first modeled using the tabulated properties available in the software [40] and then parameterized by Kramers-Kronig consistent B-spline fitting with an energy resolution of $0.1 \mathrm{eV}$, for both layer thickness and optical constants. Further parameterization using the Drude-Lorentz oscillator model was omitted because of the uncertainty in obtaining a unique solution. An example of SE data fitting with B-splines is shown in Fig. 4, indicating 


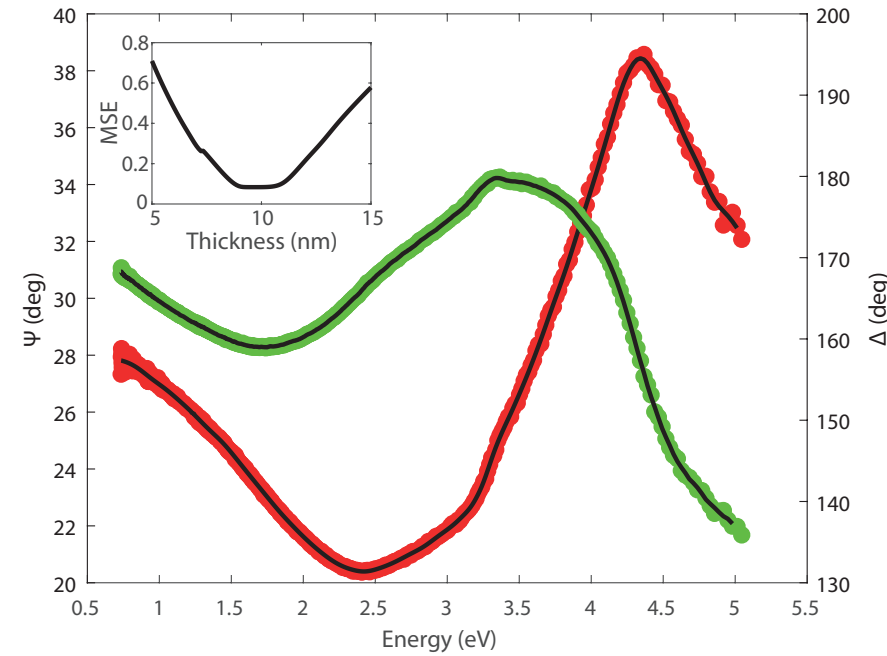

Fig. 4. Spectroscopic ellipsometry parameters $\Psi$ (red) and $\Delta$ (green). The fit obtained by the model for both parameters is indicated by the black lines. The inset shows the parameter uniqueness of the W film thickness (MSE).

a good fit. The parameter uniqueness test (see the inset) reveals not a sharp minimum, which may indicate a slight parameter correlation. From MSE, the fitted film thickness has an error margin of about $4 \%$.

Fitting the SE data for the four fabricated wafers reveals the HWALD-W-layer thicknesses of $9.6 \pm 0.4,6.3 \pm 0.2$, $5.2 \pm 0.1$ and $2.5 \pm 0.1 \mathrm{~nm}$. One should bear in mind that the SE measurements were performed on the $1 \mathrm{x} 1 \mathrm{~cm}$ area in the center of each wafer.

As expected, the growth per cycle (GPC), in the range where GPC stabilizes, is quite similar from wafer to wafer, ranging between 0.022 and $0.026 \mathrm{~nm} / \mathrm{cycle}$. A constant GPC is a characteristic of an established ALD process. Some deviations may be expected during the initial-growth stage because of the film-nucleation conditions; the latter can vary due to, for example, slightly different seed layer thicknesses.

A necessary step towards verifying validity of the proposed SE model is measuring the thickness by alternative non-optical techniques. For this, we applied high-resolution scanning electron microscopy (HR-SEM). The images were obtained in two modes by using (i) standard in-line and (ii) energy selective backscattered (ESB) detector. A filtering grid was installed in front of the ESB detector to adjust the threshold energy for enhancing the contrast and resolution; this was especially useful in application to ultra-thin films studied in this work. Fig. 5 shows a HR-SEM image of the layer stack close to the center of the wafer. The contrast difference between the layers provides a clear visualization of the HWALD W film with a thickness estimated at $10 \mathrm{~nm}$ thick, consistent with the SE-model prediction $(9.6 \pm 0.4 \mathrm{~nm})$.

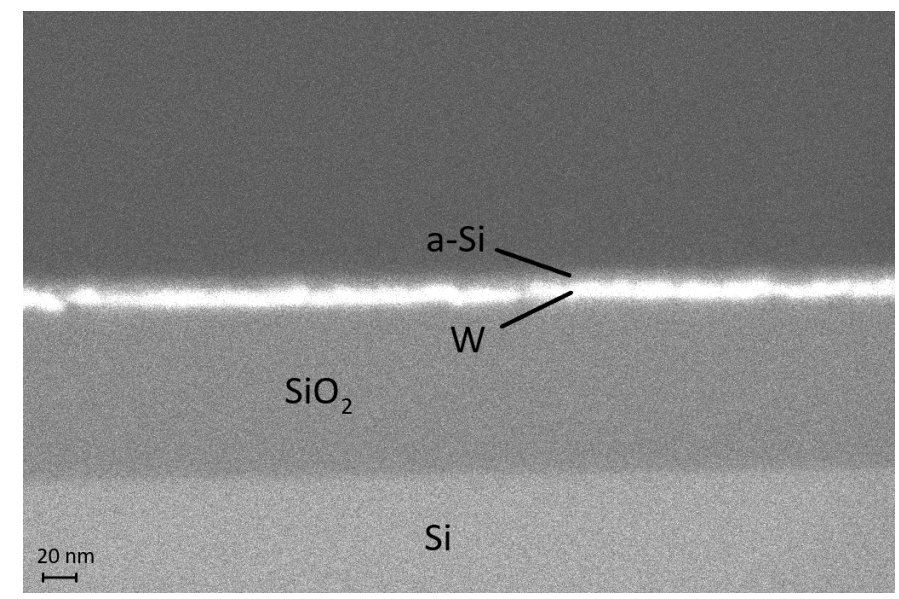

Fig. 5. High-resolution scanning electron microscopy image (obtained with an ESB detector) of the Si substrate with a roughly $100 \mathrm{~nm}$ thick thermal $\mathrm{SiO}_{2}$ layer, $10 \mathrm{~nm}$ HWALD W film (the bright layer) and a-Si capping layer.

\section{EleCtricAl MEASUREMENT RESUlts}

\section{A. Sheet resistance}

The sheet resistance of the $\mathrm{W}$ films was measured using Van der Pauw structures. Fig. 6 shows the cumulative $R_{\mathrm{sh}}$ plots of the differently-thick W films. It can be seen that $R_{\mathrm{sh}}$ increases with decreasing film thickness. The black curve indicates the magnitude of $R_{\mathrm{sh}}$ which is expected by the bulk model, i.e. for bulk $\alpha-\mathrm{W}$ resistivity $(5.6 \mu \Omega \cdot \mathrm{cm}$, [41]). The measured $R_{\mathrm{sh}}$ is higher than that of bulk $\alpha-\mathrm{W}$ on each of the wafers. The relative difference decreases towards thicker layers, indicating that the resistivity approaches the bulk value. The deviation of $R_{\mathrm{sh}}$ from the bulk value can be explained by the charge-carrier scattering effects in thin films [42], meaning reducing the carrier mobility. Scattering occurs on grain boundaries and thus increases for thinner films because of the smaller crystal grains. Additionally, the surface itself may play a role if the carrier (i.e., electron) mean free path becomes comparable with the film thickness and roughness. Finally, carrier concentration can be reduced in thin films compared to their bulks as a result of electron trapping by various defects present at both boundaries and surface.

Further, significant variations of $R_{\mathrm{sh}}$ are obtained across each wafer. For the thinner layers, the variations are presumably due to the small thickness non-uniformity of the W. As has been demonstrated in the earlier work $[18,43]$, $R_{\mathrm{sh}}$ of ultra-thin metallic layers can be extremely sensitive to very small thickness variations. The cumulative $R_{\text {sh }}$ plot of the 9.6-nm HWALD W film has been divided into two series. The variations of the $R_{\mathrm{sh}}$ are attributed mainly to a change in $\mathrm{W}$ crystallinity across the wafer. The $\mathrm{W}$ film on the bottom half of the wafer shows significantly larger $R_{\mathrm{sh}}$, compared to the top half (see the inset). 


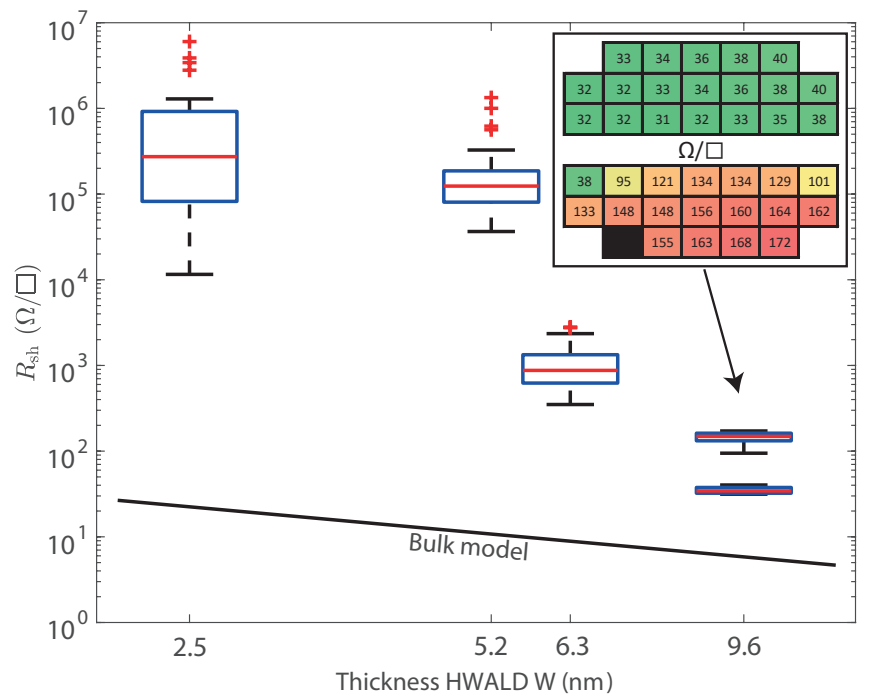

Fig. 6. The sheet-resistance variations across differently thick (as measured in the central $1 \times 1 \mathrm{~cm}^{2}$ area on each wafer) wafers. The middle-box line indicates the median, the lower and upper edges of each box show the data points statistically falling into the $25 \%$ to $75 \%$ range, respectively. The error bars indicate the most extreme data excluded from the $25 \%-75 \%$ range but still not classified as outliers. The several outliers are plotted individually using the (+) symbol. The black line indicates the theoretical sheet resistance when bulk $\mathrm{W}$ resistivity is assumed. The inset shows the variations (a.o. due to the phase change from $\alpha$ - to $\beta$-W) in the sheet resistance across the wafer for the $10 \mathrm{~nm}$ HWALD W wafer.

X-ray diffraction (XRD) measurements have been performed to determine the crystal-phases present in the two different wafer parts (see Fig. 7) of the 9.6-nm HWALD W film. The tungsten peaks within the measurement range are located at $40.2^{\circ}$ [(110) plane], 58.2 $2^{\circ}$ [(200) plane] and 73.2 [(211) plane] for $\alpha-\mathrm{W}$ [44], and at $35.5^{\circ}$ [(002) plane], $39.8^{\circ}$ [(012) plane] and $43.8^{\circ}$ [(112) plane] for $\beta$-W $[45,46]$. It is difficult to distinguish the peaks around $\sim 40^{\circ}$ due to possible overlap. However, the peaks at $35.5^{\circ}, 58.2^{\circ}$ and $73.2^{\circ}$ suggest that the top-half of the wafer contains primarily $\alpha-\mathrm{W}$, while the bottom-half of the wafer contains primarily $\beta$-W. The two different crystal phases are assumed to originate from the asymmetrical precursor flow over the wafer and the corresponding difference in the layer crystallization process as function of the growth time [17]. The resistivity of the 10 -nm-thick $\alpha-\mathrm{W}$ is estimated at $\rho=32 \mu \Omega \cdot \mathrm{cm}$, which is roughly six times larger than the bulk resistivity of $\alpha-\mathrm{W}$. The resistivity of the $10-\mathrm{nm}$-thick $\beta$-W is estimated at $\rho=121-172 \mu \Omega \cdot \mathrm{cm}$ which is at the lower margin of the bulk resistivity range reported for $\beta$-W $(100-1290 \mu \Omega \cdot \mathrm{cm}$, [47-49]). It should however be noted that the latter was obtained for sputtered W films. In contrast to that, HWALD W films may have a reduced surface roughness (due to, for example, a much lower deposition rate), keeping the impact of roughness on resistivity minimized.

Due to the variations in HWALD W film thickness across each wafer and the difficulty to measure the film thickness outside the center of the wafer, all thin-film properties in

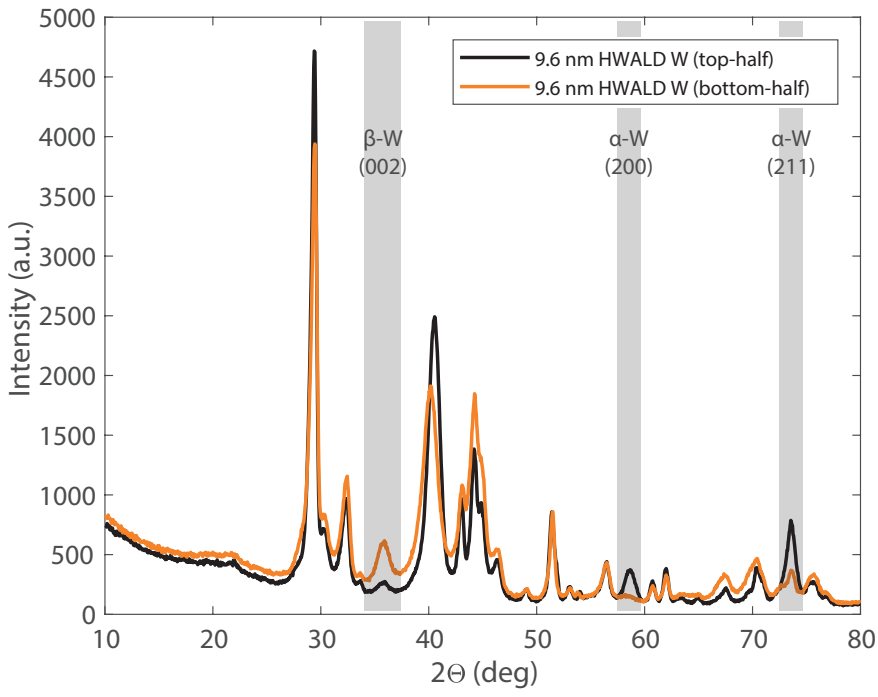

Fig. 7. $2 \Theta$ X-ray diffraction measurements of the top- and bottom-half of the $9.6 \mathrm{~nm}$ HWALD $\mathrm{W}$ wafer.

the remaining part of this work are plotted against the sheet resistance and not the film thickness.

\section{B. Contact resistance and transfer length}

To measure the contact resistance and the transfer length, the CTLM structures with 9.6-nm- and 6.3-nm-thick HWALD $\mathrm{W}$ layers were analysed. From Fig. 8a, one might suggest a slight increase of the $R_{\mathrm{C}}$ with increasing $R_{\mathrm{sh}}$, which can be explained by increased current crowding and quantum confinement in the thinner layers. However, no conclusive observation can yet be drawn due to large scattering of the data points. $L_{\mathrm{T}}$ is shown to increase sharply with decreasing sheet resistance (see Fig. 8b). This can be explained by the relative change of the magnitude of $R_{\mathrm{sh}}$ with respect to the resistance of the electrodes. For higher $R_{\mathrm{sh}}$, the current will be transferred between the electrode and the sheet over a shorter length near the edge of the electrode, thereby taking the least resistance path. One should bare in mind that the magnitude of $L_{\mathrm{T}}$ for small sheet resistance becomes such that $R_{\mathrm{el}} \gg 4 L_{\mathrm{T}}$ does not hold anymore. In future work, $R_{\text {el }}$ has to be chosen more conservatively.

\section{Temperature coefficient of resistance}

The temperature coefficient of resistance (TCR) was obtained from the Van der Pauw structures. Standard sheet resistance measurements were performed at temperatures ranging from -60 to $+200{ }^{\circ} \mathrm{C}$. The current-voltage $(I-V)$ measurements of a selected structure are shown in Fig. 9a. The negative bias range is largely excluded from the plot, to better visualize the change in $R_{\text {sh }}$ with temperature. Fig. 9b shows the evolution of $R_{\mathrm{sh}}$ as a function of temperature for structures with differently-thick $\mathrm{W}$ films. The magnitude of the TCR is determined by TCR $=1 / R_{\mathrm{sh}, T=0{ }^{\circ} \mathrm{C}} \times\left(\Delta R_{\mathrm{sh}} / \Delta T\right)$, where $\Delta R_{\mathrm{sh}}$ is the change in $R_{\mathrm{sh}}$ due to a change in temperature $\Delta \mathrm{T}$ and $R_{\mathrm{sh}, T=0}{ }^{\circ} \mathrm{C}$ is the $R_{\mathrm{sh}}$ measured at $T=0{ }^{\circ} \mathrm{C}$. The 


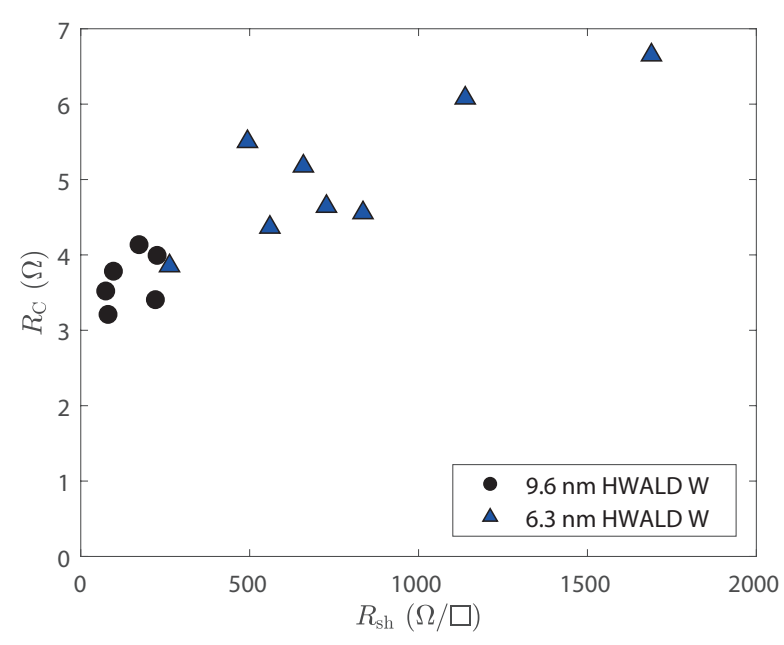

(a)

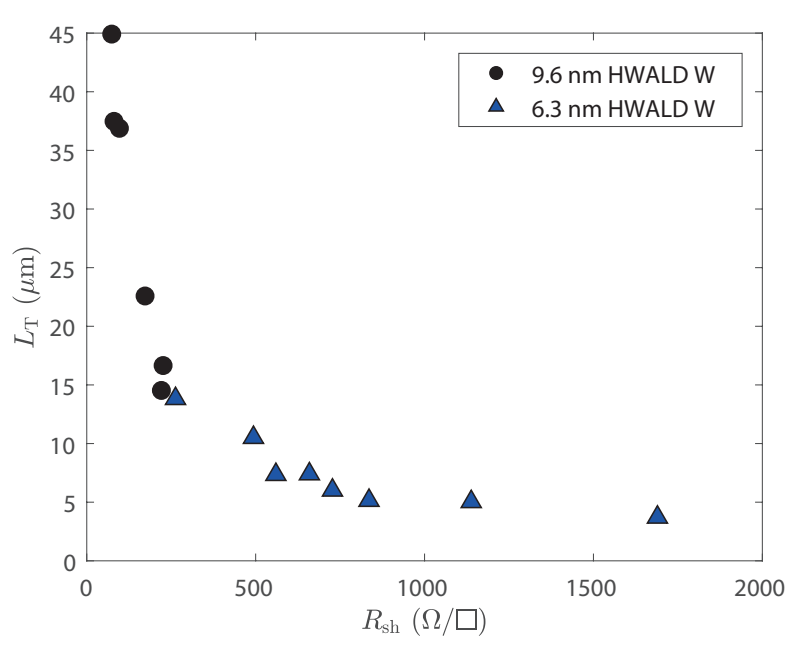

(b)

Fig. 8. The (a) contact resistance and (b) transfer length as a function of the sheet resistance. The black circles correspond to the structures with the $9.6 \mathrm{~nm} \mathrm{~W}$, while the blue triangles represent the structures having the $6.3 \mathrm{~nm}$ film.

TCR values change from positive to negative with decreasing the film thickness. Metals normally exhibit a positive TCR, which is explained by increased phonon scattering at elevated temperatures [50]. The observed negative TCR of the thinnest films can be attributed to for example the dominant hoppingtype conductance in the percolated but still not-fully-closed W film [51]. Further, for even thinner layers, a bandgap can open and a metal can start behaving as a semimetal [43]. Fig. $9 \mathrm{c}$ gives an overview of the measured TCR for structures with various film thicknesses. It can be seen that the 6.3 $\mathrm{nm}$ layer corresponds to the transition region; for this film the TCR is small and can be either positive or negative (significant variations observed across the wafer). The largest TCR of $1.1 \cdot 10^{-3}$, measured on a 9.6-nm-thick structure, is still significantly smaller than the value of the TCR for bulk W $\left(4.5 \cdot 10^{-3},[52]\right)$. It shows that thin-film effects already play a role for this thickness, though not dominantly.

\section{Field effect}

The field effect (FE) measurements were conducted by applying a constant current bias $(0.1-10 \mu \mathrm{A})$ between two adjacent Van der Pauw terminals, while measuring the voltage difference between the other two terminals as a function of the voltage $\left(V_{\mathrm{g}}\right.$, swept from -10 to $10 \mathrm{~V}$ and back) applied to the back gate. This allowed to monitor the $R_{\mathrm{sh}}$ modulation as a function of the back-gate electric field. Fig. 10a gives an example of such a $R_{\mathrm{sh}}-V_{\mathrm{g}}$ dependence, showing a little hysteresis. The FE (in $\mathrm{V}^{-1}$ ) was defined accordingly to $\mathrm{FE}=1 / R_{\mathrm{sh}}\left(V_{\mathrm{g}}=0\right) \times\left(\Delta R_{\mathrm{sh}} / V_{\mathrm{g}}\right)$, where the $\Delta R_{\mathrm{sh}}$ represents the corresponding change of $R_{\mathrm{sh}}$ due to the applied $V_{\mathrm{g}}$, and $R_{\mathrm{sh}}\left(V_{\mathrm{g}}=0\right)$ is the $R_{\mathrm{sh}}$ measured at zero $V_{\mathrm{g}}$. Fig. $10 \mathrm{~b}$ gives an overview of all FE measurements that were conducted. One can see that the FE increases with increasing sheet resistance. This is expected as the higher $R_{\mathrm{sh}}$ can be directly related to the lower electron concentration [43]. The devices with the lowest sheet resistance exhibit near zero FE. The largest field effect of $\sim 4.6 \cdot 10^{-4} \mathrm{~V}^{-1}$ is larger than the typical magnitude of the field effect in metals $\left(\sim 10^{-5}[5-10]\right)$, but smaller than that reported for ultra-thin TiN films [43]. However, varying definitions of the field effect complicate a fair comparison. Further, especially for metals, the field effect depends strongly on layer thickness. Dependence of the FE on temperature, varied between 40 and $160{ }^{\circ} \mathrm{C}$, showed no conclusive trend.

\section{CONCLUSIONS}

Thin $(2.5-10 \mathrm{~nm})$ tungsten films obtained by the novel HWALD technique have been characterized for the first time in terms of their electrical performance. The developed SE model, verified by HR-SEM, could adequately describe the film thickness. The XRD analysis indicated the formation of both $\alpha-$ and $\beta$-phase tungsten in the thickest film. Clear dependence of the sheet resistance, TCR and FE on the layer thickness has been demonstrated. The remarkable transition from positive to negative TCR at around $6.3 \mathrm{~nm}$ of the thickness has been observed. A field effect of approx. $\sim 4.6 \cdot 10^{-4} \mathrm{~V}^{-1}$ has been measured, which is larger than the typical value obtained for metals so far.

\section{REFERENCES}

[1] O. Sneh, R.B. Clark-Phelps, A.R. Londergan, J. Winkler, and T.E. Seidel, "Thin film atomic layer deposition equipment for semiconductor processing", Thin Solid Films, Volume 402, Issues 1-2, pp. 248-261, 2002.

[2] A. Paranjpe, S. Gopinath, T. Omstead, R. Bubber, "Atomic layer deposition of $\mathrm{AlO}_{x}$ for thin film head gap applications", Journal of the Electrochemical Society, Volume 148, Issue 9, pp. G465-G471, 2001.

[3] T. Luoh, C.-T. Su, T.-H. Yang, K.-C. Chen, C.-Y. Lu, "Advanced tungsten plug process for beyond nanometer technology", Microelectronic Engineering, Volume 85, Issue 8, pp. 1739-1747, 2008.

[4] F. Papadatos et al, "Low resistivity tungsten for $32 \mathrm{~nm}$ node MOL contacts and beyond", Microelectronic Engineering, Volume 92, pp. 123125, 2012.

[5] H.L. Stadler, "Changing Properties of Metals by Ferroelectric Polarization Charging", Physical Revies Letters, Volume 14, Issue 24, pp. 979-981, 1965. 


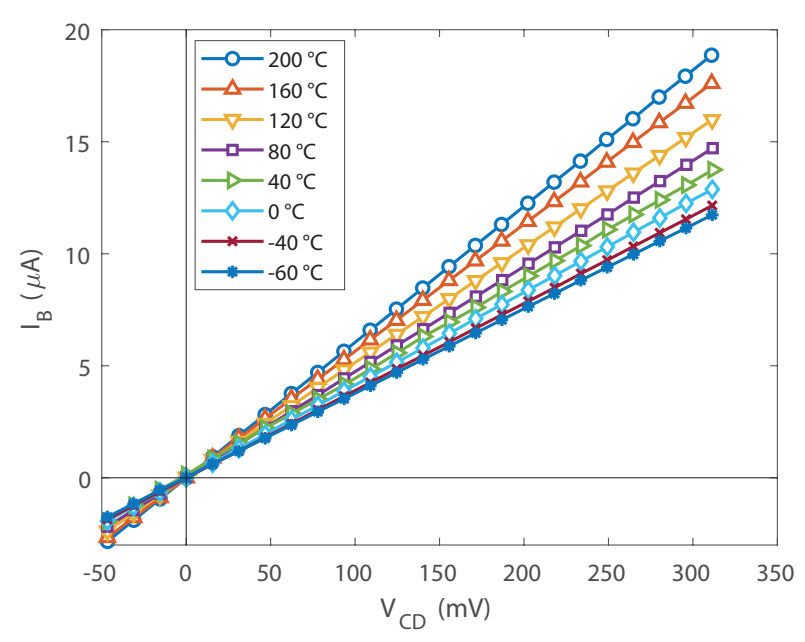

(a)

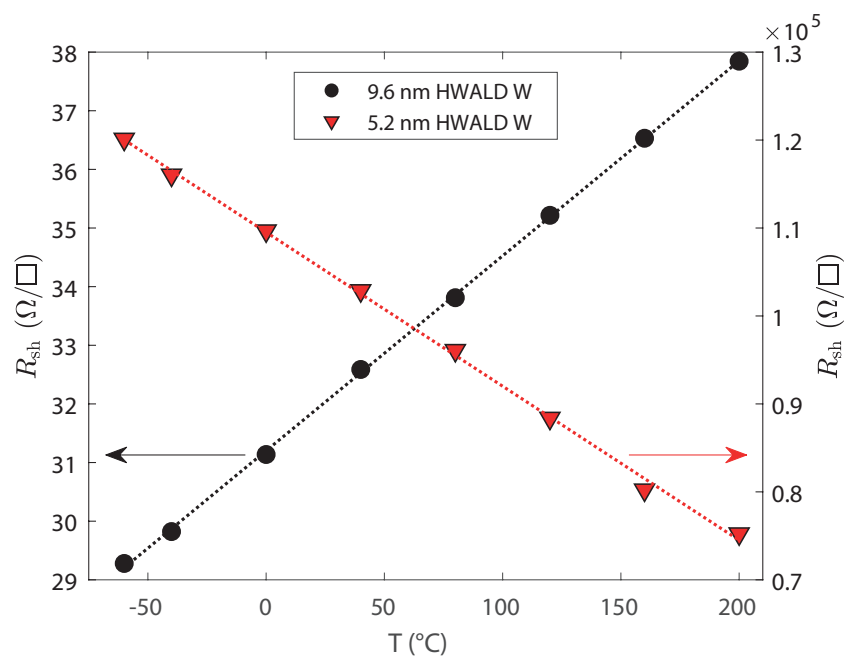

(b)

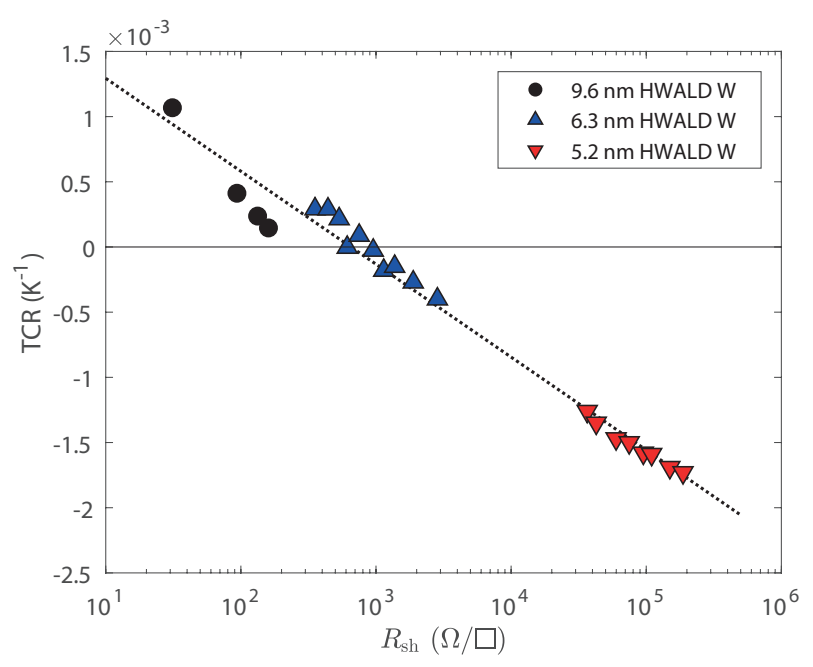

(c)

Fig. 9. Temperature dependent sheet resistance measurements. (a) IV curves of a selected 5.2-nm-thick structure. (b) Sheet resistance of selected 9.6$\mathrm{nm}-$ and 5.2-nm-thick structures showing a positive and negative TCR, respectively. (c) The cumulative TCR versus sheet resistance plot for various film thicknesses (wafers) and structure positions on each wafer.

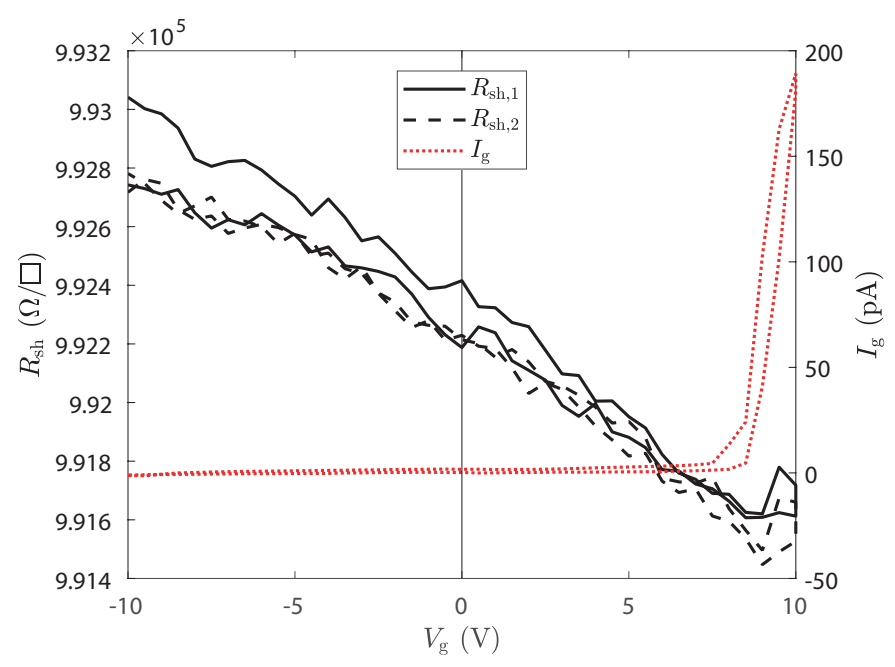

(a)

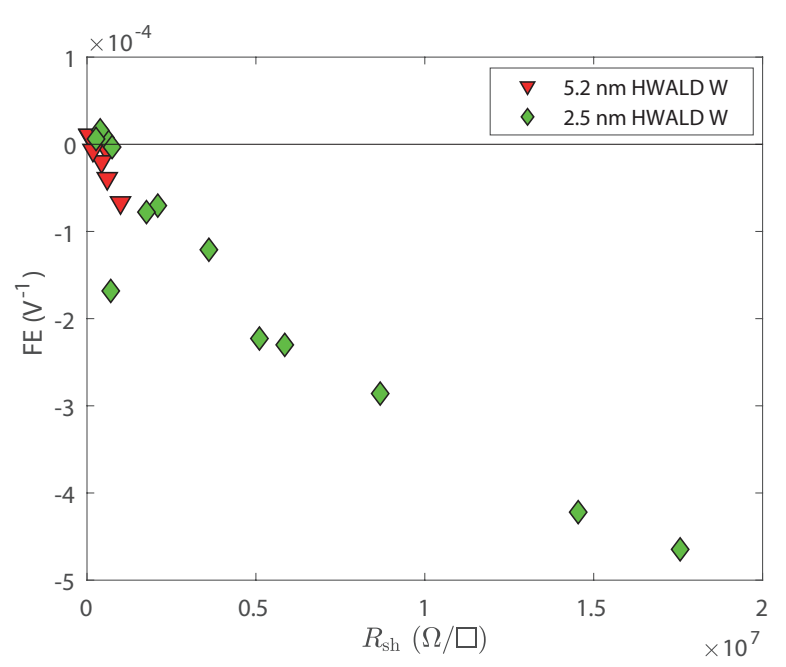

(b)

Fig. 10. (a) The change of the sheet resistance of a selected 1-nm-thick structure while sweeping the back-gate voltage $\mathrm{V}_{g}$ from $-10 \mathrm{~V}$ to $10 \mathrm{~V}$ and backwards; the flat curve with a sharp increase at $\sim 8 \mathrm{~V}$ corresponds to the gate leakage current $\mathrm{I}_{g}$. (b) The observed field effect as a function of the sheet resistance, for the indicated film thicknesses (wafers) and various structure positions on each wafer.

[6] H.J. Juretschke, "Influence of the surface scattering of electrons on the metallic field effect in thin layers", Surface Science, Volume 5, Issue 1, pp. 111-119, 1966.

[7] E.C. McIrvine, "Influence of boundary scattering on the metallic field effect", Surface Science, Volume 5, Issue 2, pp. 171-178, 1966.

[8] A. Berman and H.J. Juretschke, "Origin of the electric field effect in silver", Applied Physics Letters, Volume 18, Issue 10, pp. 417-418, 1971.

[9] R. Dimmich and F. Warkusz, "A simple model of the electric field effect in thin metallic films", Thin Solid Films, Volume 79, Issue 2, pp. 173$184,1981$.

[10] R. Dimmich and F. Warkusz, "The metallic field effect in thin polycrystalline films", Thin Solid Films, Volume 135, Issue 1, pp. 43-50, 1986.

[11] S.M. George, "Atomic layer deposition: an overview", Chemical reviews, vol. 110, pp. 111-131, 2009.

[12] B.S. Lim, A. Rahtu and R.G. Gordon, "Atomic layer deposition of transition metals", Nature Materials, Volume 2, Issue 11, pp. 749-754, 2003. 
[13] V.V. Afanas'ev, J.M.M. de Nijs, P. Balk and A. Stesmans, "Degradiation of thermal oxide of the $\mathrm{Si} / \mathrm{SiO}_{2} / \mathrm{Al}$ system due to vacuum ultraviolet irradiation", Journal of Applied Physics, Volume 78, Issue 11, pp. 64816490, 1995.

[14] A.Y. Kovalgin, M. Yang, S. Banerjee, R.O. Apaydin, A.A.I. Aarnink, S. Kinge, and R.A.M. Wolters, "Hot-Wire Assisted ALD: A study powered by in situ spectroscopic ellipsometry", Advanced Materials Interfaces, Volume 4, Issue 18, 1700058, 2017.

[15] M. Yang, A.A.I. Aarnink, A.Y. Kovalgin, R.A.M. Wolters and J. Schmitz, "Hot-wire assisted ALD of tungsten films: In-situ study of the interplay between CVD, etching, and ALD modes", Physica Status Solidi (A) Applications and Materials Science, Volume 212, Issue 7, pp. 1607-1614, 2015.

[16] M. Yang, A.A.I. Aarnink, A.Y. Kovalgin, D.J. Gravesteijn, R.A.M. Wolters and J. Schmitz, "Comparison of tungsten films grown by CVD and hot-wire assisted atomic layer deposition in a cold-wall reactor", Journal of Vacuum Science and Technology A: Vacuum, Surfaces and Films, Volume 34, Issue 1, 01A129, 2016.

[17] M. Yang, A.A.I. Aarnink, R.A.M. Wolters, J. Schmitz and A.Y. Kovalgin, "Effects of oxygen, nitrogen and fluorine on the crystallinity of tungsten by hot-wire assisted ALD", ECS Journal of Solid State Science and Technology, Volume 6, Issue 12, pp. P839-P844, 2017.

[18] M. Yang, A.A.I. Aarnink, J. Schmitz and A.Y. Kovalgin, ”Low-resistivity $\alpha$-phase tungsten films grown by hot-wire assisted atomic layer deposition in high-aspect-ratio structures", Thin Solid Films, Volume 646, pp. 199-208, 2018.

[19] M. Yang, A.A.I. Aarnink, J. Schmitz and A.Y. Kovalgin, 'Inherently area-selective hot-wire assited atomic layer deposition of tungsten films", Thin Solid Films, Volume 649, pp. 17-23, 2018.

[20] K. van der Zouw, A.A.I. Aarnink, J. Schmitz and A.Y. Kovalgin, "Electrical characterization of hot-wire assisted atomic layer deposited tungsten films", Proc. IEEE ICMTS 2019, Mar. 2019, pp.48-53.

[21] L.J. van der Pauw, "A method of measuring specific resistivity and Hall effect of discs of arbitrary shape", Philips Research Reports, vol. 13, no. 1, pp. 1-9, 1958.

[22] L.J. van der Pauw, "A method of measuring the resistivity and Hall coefficient on lamellae of arbitrary shape", Philips Technical Review, vol. 20, no. 8, pp. 220-224, 1958/59.

[23] W. Versnel, "Analysis of symmetrical Van der Pauw structures with finite contacts", Solid-State Electronics, Volume 21, pp. 1261-1268, 1978.

[24] A.J. Willis and A.P. Botha, "Investigation of ring structures for metalsemiconductor contact resistance determination", Thin Solid Films, vol. 146, pp. 15-20, 1987.

[25] J.H. Klootwijk and C.E. Timmering, "Merits and limitations of circular TLM structures for contact resistance determination for novel IIIV hbts", Proc. IEEE 2004 Int. Conference on Microelectronic Test Structures, vol. 17, pp. 247-252, 2004.

[26] G.S. Marlow and M.B. Das, "The effects of contact size and non-zero metal resistance on the determination of specific contact resistance", Solid-State Electronics, Volume 25, Issue 2, pp. 91-94, 1982.

[27] M. Ahmad and B.M. Arora, "Investigation of AuGeNi contacts using rectangular and circular transmission line model", Solid-State Electronics, Volume 35, Issue 10, pp. 1441-1445, 1992.

[28] H. Fujiwara, "Spectroscopic Ellipsometry: Principles and Applications", John Wiley \& Sons, 2007.

[29] H.G. Tompkins and E.A. Irene, "Handbook of Ellipsometry", William Andrew Publishing, 2005.

[30] G.E. Jellison and F.A. Modine, "Optical functions of silicon between 1.7 and $4.7 \mathrm{eV}$ at elevated temperatures", Physical Review B, Volume 27, Issue 12, pp. 7466-7472, 1983.

[31] C.M. Herzinger, B. Johs, W.A. McGahan, J.A. Woollam and W. Paulson, "Ellipsometric determination of optical constants for silicon and thermally grown silicon dioxide via a multi-sample, multi-wavelength, multi-angle investigation”, Journal of Applied Physics, Volume 83, Issue 6, pp. 3323-3336, 1998.

[32] S. Roberts, "Optical properties of Nickel and Tungsten and their interpretation according to Drude's formula", Physical review, Volume 114, Issue 1, pp. 104-115, 1958.

[33] J.H. Weaver, C.G. Olson and D.W. Lynch, "Optical properties of crystalline tungsten, Physical Review B, Volume 12, Issue 4, pp. 12931297, 1975.

[34] L.F. Mattheis, "Fermi Surface in Tungsten", Physical Review, Volume 139, Issue 6A, pp. 1893-1904, 1965.
[35] P. Romaniello, P.L. de Boeij, F. Carbone and D. van der Marel, "Optical properties of bcc transition metals in the range $0-40 \mathrm{eV}$, Physical Review B, Volume 73, pp. 075115, 2006.

[36] P. Gravier, G. Chassaing and M. Sigrist, "Optical properties of thin films of bcc transition metals", Thin Solid Films, Volume 57, pp. 93-98, 1979.

[37] J.E. Nestell Jr., R.W. Christy, M.H. Cohen and G.C. Ruben, "Structure and optical properties of evaporated films of the $\mathrm{Cr}$ - and V-group metals", Journal of Applied Physics, Volume 51, pp. 655-660, 1980.

[38] D. Davazoglou, G. Pallis, V. Psycharis, M. Gioti and S. Logothetidis, "Structure and optical properties of tungsten thin films deposited by pyrolysis of $\mathrm{W}(\mathrm{CO})_{6}$ at various temperatures", Journal of Applied Physics, Volume 77, pp. 6070-6072, 1995.

[39] A.G. Deineka, A.A. Tarasenko, L. Jastrabik, D. Chvostova and J. Bousek, "An ellipsometric study of W thin films deposited on Si", Thin Solid Films, Volume 339, pp. 216-219, 1999.

[40] E.D. Palik (Ed.), Handbook of Optical constants of Solids, 1, Academic Press, San Diego, pp. 358-368, 1998.

[41] R.A. Serway, Principles of physics (2nd ed.), Fort Worth, Texas; London: Saunders College Pub. p.602

[42] G.P. Zhigal'skii and B.K. Jones, The Physical Properties of Thin Metal Films, Taylor and Francis, New York, NY, USA, 2003.

[43] H. Van Bui, A.Y. Kovalgin, J. Schmitz, and R.A.M. Wolters, APPLIED PHYSICS LETTERS, Volume 103, Issue 5, pp. 051904, 2013.

[44] F. Allen, O. Kennard, D. Watson, L.Brammer, A. Orpen and R. Taylor, International Tables for Crystallography (Kluwer Academic, Fordrecht 1995), Vol. C.

[45] E. Lassner and W.D. Schubert, Tungsten: Properties, Chemistry, Technology of the Elements, Alloys, and Chemical Compounds (Springer Science \& Business Media, New York, 1999)

[46] A. Bartl, "Fundamentals of NS-tungsten powder manufacture" Ph.D. thesis (Tu Vienna, 1997)

[47] P.M. Petroff, T.T. Sheng, A.K. Sinha, G.A. Rozgonyi, and F.B. Alexander, "Microstructure, growth, resistivity, and stresses in thin tungsten films deposited by rf sputtering", Journal of Applied Physics, Volume 44, pp. 2545, 1973.

[48] Q. Hao, W. Chen and G. Xiao, "Beta $(\beta)$ tungsten thin films: Structure, electron transport, and giant spin Hall effect", Applied Physics Letters, Volume 106, pp. 182403, 2015.

[49] P.M. Petroff and W.A. Reed, "Resistivity behavior and phase transformations in $\beta$-W thin films", Thin Solid Films, Volume 21, Issue 1, pp. 73-81, 1974.

[50] N.W. Ashcroft and N.D. Mermin, "Solid State Physics", Harcourt Brace College Pub., New York, 1976.

[51] H. Bottger and V.V. Bryksin, "Hopping conductivity in ordered and disordered solids (I)", Physica status solidi B, Volume 78, Issue 9, pp. 9-56, 1976.

[52] R.A. Serway and J.W. Jewett, Principles of Physics: A Calculus-Based Text (5th ed.), Belmont, CA: Brooks/Cole, 2013 